\title{
Managing Virtual Team Work in IT Projects: Survey
}

\author{
http://dx.doi.org/10.3991/ijac.v7i4.4018 \\ Hosam El-Sofany ${ }^{1}$, Hassan Alwadani ${ }^{2}$, and Amer Alwadani ${ }^{3}$ \\ ${ }^{1}$ Cairo Higher Institute, Cairo, Egypt \\ ${ }^{2}$ Arab East Colleges for Graduate Studies, Riyadh, Kingdom of Saudi Arabia \\ ${ }^{3}$ University of Dayton, Ohio State, USA
}

\begin{abstract}
- the nature of managing team work has changed significantly because of changes in organizations and the nature of the work they do. Organizations have become more distributed across geography and across industries. Relationships between people inside an organization and those previously considered outside (customers, suppliers, managers of collaborating organizations, other stakeholders) are becoming more important. Organizations have discovered the value of collaborative work. When implementing IT projects in a global environment, a popular strategy is the establishment of virtual teams. The objective of this paper is to examine what competence an IT project manager has to have to lead a virtual IT team successfully. The question is approached from the perspective of competences presented in the IPMA (International Project Management Association) competence baseline, or The Eye of Competences. Results from research, as well as a survey conducted, highlighted that both soft and hard skills are needed for the optimal IT project manager to lead a virtual team. To be successful in this area it is not sufficient to be outstanding in technical competences. Soft skills like communication, teamwork and leadership are no less essential. The competences scoring highest in the survey were: clarity of objectives, leadership \& project management, communication, reliability \& efficiency, teamwork and quality management.
\end{abstract}

Index Terms-Project management, Virtual teams, Software teams

\section{INTRODUCTION}

In recent years, companies have increasingly turned to virtual teams as a means of connecting and engaging geographically dispersed workers, lowering the costs associated with global collaboration, and enabling greater speed and adaptability. Virtual teams have the strong advantage of gathering the best people for a specific task independent of their geographical location in a sort of 'just in time talent' approach.

The software industry is no exception. It has been impacted by globalization and this trend continues today. These changes have been coupled with the availability, in large numbers, of qualified software engineers located in low cost economies, who are capable and willing to undertake outsourced and offshore software development. Virtual teams, while offering many benefits, also pose a number of challenges. One of these challenges is to develop effective global leaders.
In this changing environment, what competence has an IT project manager to excel in to be able to take on these challenges? What soft and hard skills do they have to focus on?

In this paper the above mentioned question are approached from the perspective of competences presented in the IPMA competence baseline, or The Eye of Competences. This study, however, only focuses on the technical and behavioral categories, leaving the contextual category untouched [1].

\section{RELATED WORKS}

In [2] the research was focused on findings from literature view is used to develop a Project Management Cultural Framework. This framework accounts for different cultures in international project teams and identifies culture and communication as core issues framed by a project management methodology, organizational systems, processes and infrastructure as well as the external environment and stakeholders. Additionally, the framework suggests that an appropriate management of inter-cultural teams poses specific challenges to project managers and organizations striving to build successful project teams.

In [3], the main outcome is that a combination of soft and hard is needed to lead virtual teams in IT projects. Technical competence is not sufficient to be successful in this field. In most cases there was no significant correlation between two parameters; usually only a slight or no correlation. The exception was a correlation between a sense of isolation and the team never meeting face to face. Also a correlation was found between some competences and the role in teams.

In [4], the researchers introduced a study to develop a cross-cultural communication model that can be applied by companies that communicate with employees from different cultures. Their aim is to identify the steps that leaders of organizations competing in a global environment should consider when communicating to different cultures. the 4 C's Global Leadership Model is introduced to demonstrate how organizational leaders can create innovation in a global environment by managing conflict, communication, creativity, and connectivity.

In [5], the author attempted to look at the abovementioned factors from a human perspective, and the result from his study that the greatest challenges faced by virtual teams are direct communication, creating group dynamics and trust. The four key success factors are: 
communication, culture, technical competence and a sense of leadership and direction delivered face to face early

Case study: the Icelandic situation:

A project in Information Technology: The project consists of four members with the Dutch manager two in Iceland and one in Denmark and one in the UK. Project duration 6 months. The team was working in the form of virtual team from different places and different time. The team faced a problem in determining the scope of the project and its objectives and spent more time and space is not clear.

Director Dutch decided to direct and important meeting in Iceland to learn more about the team members from near and identify goals and objectives and was the launch of the project.

The virtual team continues the project via Skype in the remaining period for a period of between 25 to 30 minutes a day until they reached the main objective of the project deliver high quality product.

Success factors:

- Organized kick off meeting for the project.

- Clear directions, and the scope was narrowed down.

- The team is using authorized methods, like Scrum.

- The team has daily virtual meetings for updates.

- Face to face meetings in every four weeks.

- Invested time in building relationship and trust.

- Issues are brought up and discussed right away.

- The project manager is working on solutions for the issues.

- Unsuccessful factors:

- The project owner is very occupied in other assignments.

- The project owner is not sharing enough knowledge with the team [5].

In [6], the author examined the impact of the workforce on Human Resource Management either locally or internationally also it revealed the various factors driving globalization in the workforce and the issues and challenges that confront the Human Resource Management in the global markets, and also the benefits of globalization $\mathrm{Hu}-$ man Resource Information System (HRIS) to the Human Resource (HR) department.

Distributed/virtual project teams

In the IPMA competence baseline ICB 3.0 (2006), a team is defined as a "group of people who work together to realize specific objectives." However, it does not define virtual teams separately. The IPMA Eye of Competence is divided in three categories listed here below, as shown in Figure 1.

Behavioral Competences: Leadership, engagement and motivation, self-control, assertiveness, relaxation, openness, creativity, results of orientation, efficiency, consultation, negotiation, conflict and crisis, reliability, value appreciation, and ethics.

Technical Competences: Project management success, interested parties, project requirements and objectives, risk and opportunity, quality, project organization, teamwork, problem resolution, project structures, scope and deliverables, time and project phases, resources, cost and finance, procurement and contracts, changes, control and reports, information and documentation, communication, start-up, and closeout.

Contextual Competences: Project orientation, program orientation, portfolio orientation, project program and portfolio implementation, permanent organization, business, systems products and technology, personnel management, health, security, safety and environment, finance, and legal.

In [7], the author states that: "Virtual teams can be defined as a group of people with a shared goal who fulfill their roles with little or no time spent meeting face to face ,"

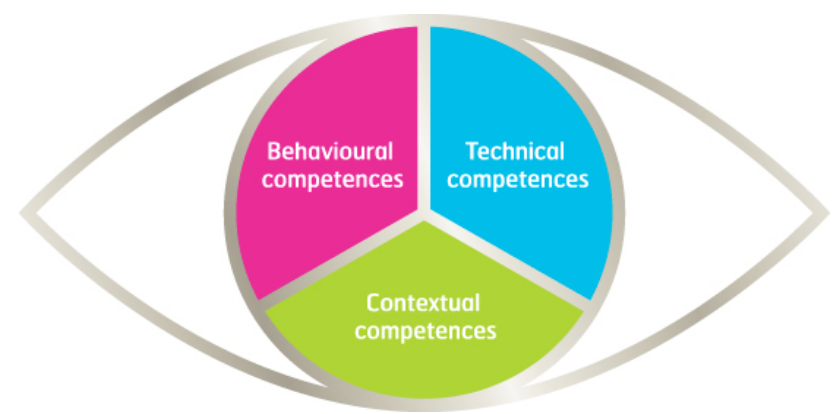

Figure 1. IPMA Eye of Competence [7]

Many other definitions exist for distributed/virtual teams. Most of these define distributed teams as teams where the bulk of the communication is done with the aid of information technology. Lipnack and Stamps (1997) define distributed teams as groups which work across space, time and organizations, and where communication is through information technology. Some define distributed teams as those who never meet whilst others define them as teams which meet very rarely [8]. Some scholars also differentiate between globally distributed teams and those who work within the borders of one country. Typical problems in globally distributed teams may be different from those in teams who work within the same borders, i.e. language problems, different cultural backgrounds and large distances $[8,9]$.

Global virtual teams are different from intra-national virtual teams in that they are not only separated by time and space, but differ in national, cultural, and linguistic attributes. According to Wheatley and Wilemon (1999), a global team is defined as a team that is comprised of individuals located in many different countries or geographic areas. Team members differ in their functionality, which adds complexity to the group dynamics. The main idea behind this concept is that people are both geographically and functionally dispersed. Conversely, traditional teams consist of people that work collaboratively, but usually these are located in one location and may or may not be functionally different. In global virtual teams, the barriers can be larger because of national differences [10].

A virtual team (also known as a geographically dispersed team or GDT) is a group of individuals who work across time, space and organizational boundaries with links strengthened by webs of communication technology. Other issues that emerged in the pilot survey regarded the wording of the questions as some of them turned out to be too complicated.

Virtual team leaders: The challenges that virtual team leaders face are immense. Many report that they feel as if they are the "glue" that holds their teams together. They 
have to establish trust in an environment with little or no face-to-face contact or feedback. These challenges necessitate the development of an additional set of competencies that complement the skills for leading traditional teams [11].

Ingason and Jónasson (2009) argue that the project manager should have hard skills and technical skills as well as soft skills like those that relate to leadership, motivation, group dynamics, interpersonal communication, culture, and ethics, to manage the project effectively. Further, soft skills will help in managing people from diverse cultures and managing conflicts among the teammembers. Finally, they prove that although technical ability is an important skill for the project manager, a strong focus should be given to leadership strategy and skills.

Soft skills are subjective and may or may not be true. They are often associated with personal attributes and character. Some examples include:

- Strong communication skills

- Time management

- Organizational skills

- Good with children

- Reliable, responsible, committed

Hard skills are specific, teachable abilities that are based in fact. Some examples include:

- Project requirements \& objectives are clear

- Language.

- Scope \& deliverables are well defined

- Quality

- Conflict \& crisis handling

The challenges faced by virtual managers are different from their non-virtual counterparts because they must deal with feelings of isolation as well as communication difficulties. Trust, a critical factor for effective virtual working, is harder to build, while technology is both an aid and barrier to effective communication. Psychological issues around cultural diversity emerge as virtual workers become distanced from their organization [12].

The outcome presented in [12] is confirmed that communications, building and maintaining trust, selecting the right people, developing the team and an outcome-based approach to performance management are all important for effective virtual work processes. Of these, communication and trust were most critical with effective virtual managers excelling in these areas.

\section{A. Leading IT projects/software development and globalization}

IT technologies change at a rapid pace and the IT project manager can rarely be expected to learn the technical aspects. However, an IT project manager must have the ability to recruit and form the team to manage the project. He should have effective leadership ability i.e. vision and the ability to influence both the stakeholders and the project team.

There is a distinct difference between an IT and non-IT project like engineering, construction, manufacturing, etc. An IT project is never standardized and comprises a variety of activities defined as follows:
1. IT development is very rapid. As a consequence, any prediction of IT projects and technologies are quite difficult.

2. There is no yardstick or standard to measure the economic viability of IT projects and activities [13].

During the 90s, the trend was for software companies to outsource software development to a third party or subsidiaries based in Ireland, and India [13].

In recent years, however, the focus has shifted away from Ireland and Israel to Eastern Europe, Latin America, and the Far East, where China and India are the most popular locations. The number of organizations globalizing their information systems development continues to rise. This means that globally distributed software development is set to continue to have a significant impact on the way the software industry operates for the foreseeable future [14]

Technical skills alone are not sufficient for the success of any IT project in today's distributed and complex workplace. IT projects are dispersed all over the globe and now the project manager must acquire the soft skills and leadership ability to lead the project in order to achieve the measurable organizational objectives [16].

Research on critical success factors in IT project management by Thite (1999) focuses on the importance of non-technical factors, such as leadership. He describes the three dimensions, i.e. the self, the subordinate and superior perspective of successful leadership characteristics in IT project management. He argues that leadership is tangible and characterized by organizational catalysts, intellectual stimulation, behavioral charisma and contingent reward behaviors for enhanced leadership effectiveness [16].

\section{CONTRIBUTION}

The purpose of the survey was to try to discover what competence IT project managers have to have to lead a virtual team successfully, e.g. what type of hard and soft skills are needed. The guidelines for competence are taken from the IPMA Eye of Competence, i.e. the technical and behavioral sectors. However, the contextual was not included.

We have designed and implemented a questionnaire. The questionnaire was divided into three parts. The first part focused on background information, such as gender, experience with international projects and international training. Most of the questions in this section were traditional background questions, but to maintain anonymity it was necessary to limit this section.

In the second part, questions were asked about the last project that the individual participated in and included questions like the role in the team, team size, the number of organizations in the project, the number of locations and the maximum time difference between locations, frequency of communication, communication media, implementation of the start up/kick off and also whether some team building was done. Finally, participants were asked whether they had noticed problems in the last project. They got a list of 14 items that are often referred to as a problem in distributed teams.

The third and last section focused on the project manager competences. Participants were asked to prioritize and rate the technical and behavioral competence according to importance. The participants were also asked to 
select from a list what they considered to be the main challenges of working in a virtual team.

\section{RESUlTS}

The main outcome from the survey is that a combination of soft and hard is needed to lead virtual teams in IT projects. Technical competence is not sufficient to be successful in this field. In most cases there was no significant correlation between two parameters; usually only a slight or no correlation. The exception was a correlation between a sense of isolation and the team never meeting face to face. Also a correlation was found between some competences and the role in teams.

\section{A. Survey finding}

We selected 31 participants, 24 were male and 7 female. Educational level was high; 14 participants had cross-cultural training and 17 had no cross-cultural training. The average of VT projects that the participants participated from 6-10 VT projects. There were only Five nationalities: Saudis, Egyptian, Gordian, Pakistani, Indian .but the locations were five, i.e. the Saudi Arabia, Egypt, the US, Pakistan, UK and Indian.

$74 \%$ percent of the participants were regular team members in their project; the rest, or $26 \%$, were project managers. The average team size was around 12 members, with the most common size being 4-6 individuals. $65 \%$ of the projects had 9 or more members. Average project time was 6 months but the most common time was 6-12 months. Most of the projects had 2 languages or $54.2 \%$ and no project had more than four languages. The average number of organizations involved was 2 . In most of the projects, three organizations were involved or $39 \%$. The average number of project locations was 2.5 ; most had two or three locations. On average, the largest time zone difference was 4 hours; most common were 2 hours differences.

Most of the teams had little direct communication, face to face, with $10 \%$ never meeting and $90 \%$ meeting once during a project's duration. Phone calls or Skype calls, Mails, Team Viewer, video calls were often the main mode of communication. The frequency of team meeting was rather high: $94 \%$ had team meetings weekly on internet and some of projects meets on daily.

\section{B. Problems occurred in the projects}

Figure 2, presents the main problems encountered in the project teams. Lack of clear objectives for the project and technical problems were the most common problems, but time difference, different public holidays, and recognizing the need for support was also reported. Very few encountered problems due to no commitment, cultural differences, lack of trust or lack of leadership. No one encountered problems due to religious views.

The elements that participant found challenging with working in virtual team are displayed in Figure 3.

There was a significant correlation between those participants who reported a sense of isolation and the team never meeting face to face, or $62.5 \%$ of the participants.

The ranking of behavioral competence when the participants were asked to rate each separately is shown in Figure 4.

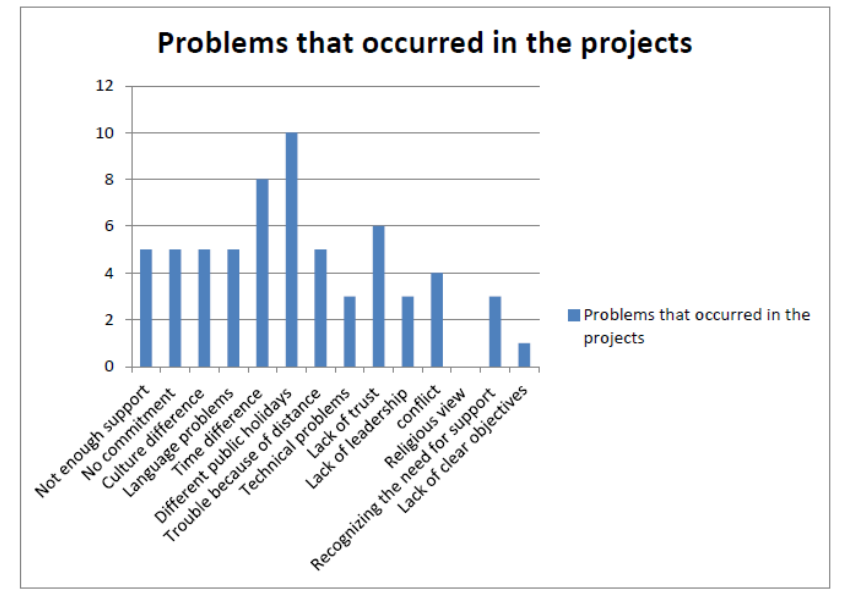

Figure 2. Problems that occurred in the projects

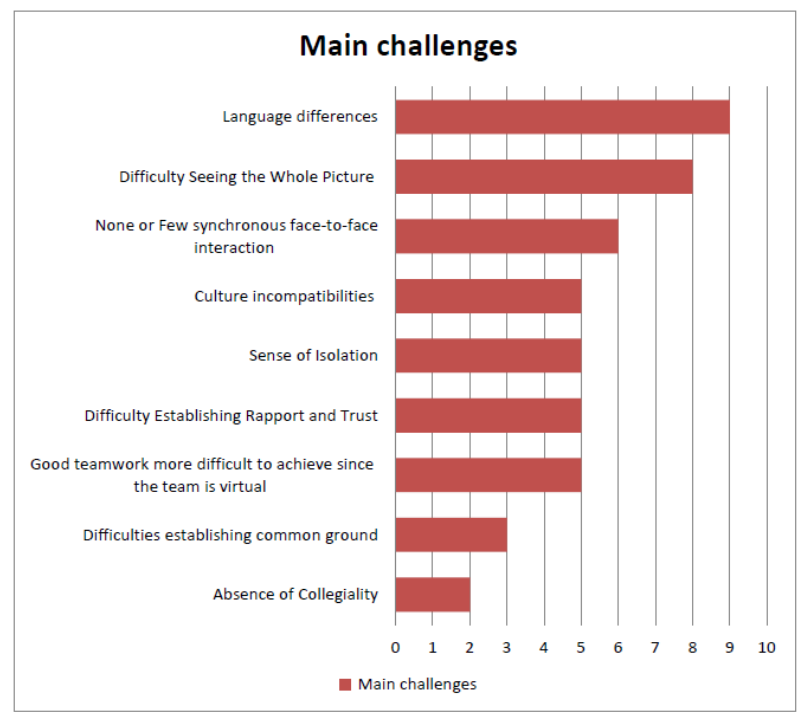

Figure 3. Elements that participant found challenging

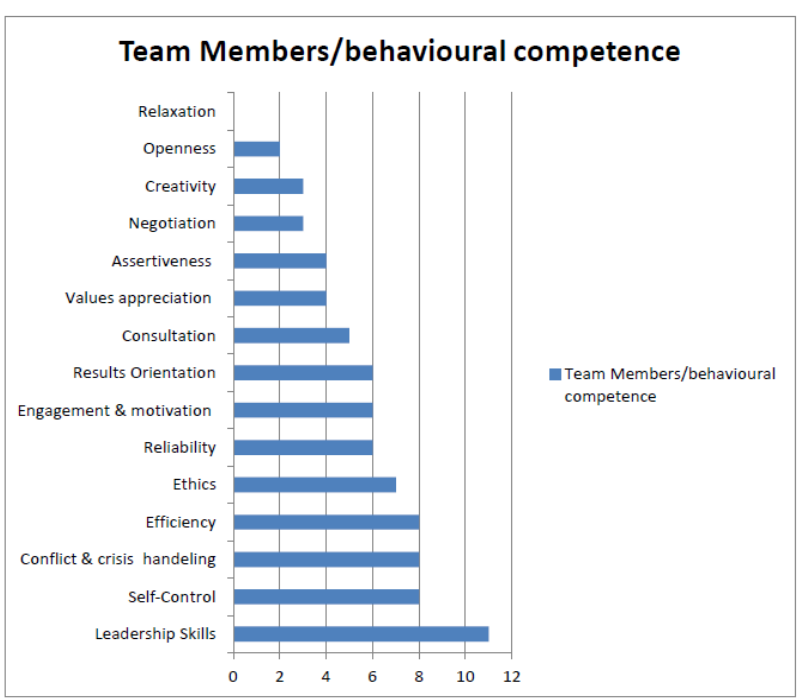

Figure 4. Correlation between importance of behavioral competence and role in team 
The rating of the technical competence, i.e. when participants were asked to rate each separately is shown in Figure 5.

Below on Figure 6 the ten competences that scored highest in participant rating are listed. The Table 1 shows how these competences are categorized into technical and behavioral competences on the one hand, and hard and soft skills on the other hand.

Figure 7, shows the result from the survey, division between technical and behavioral competences on the one hand, and hard and soft skills on the other hand shown in Figure 8 .

\section{DISCUSSION}

This paper has studied the project manager competences needed to lead an IT project in a distributed environment. It has looked at what combination of behavioral and technical competence might be needed. First, this topic was discussed from the perspective of existing literature, and then from the outcome of the survey that was conducted within a IT projects, which is a project driven company using virtual teams.

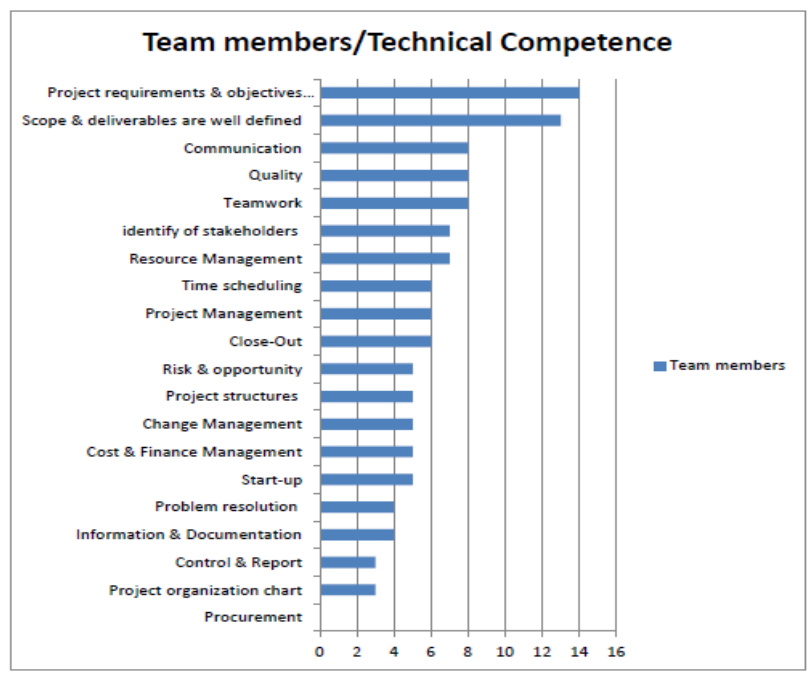

Figure 5. Correlation between importance of technical competence and role in team

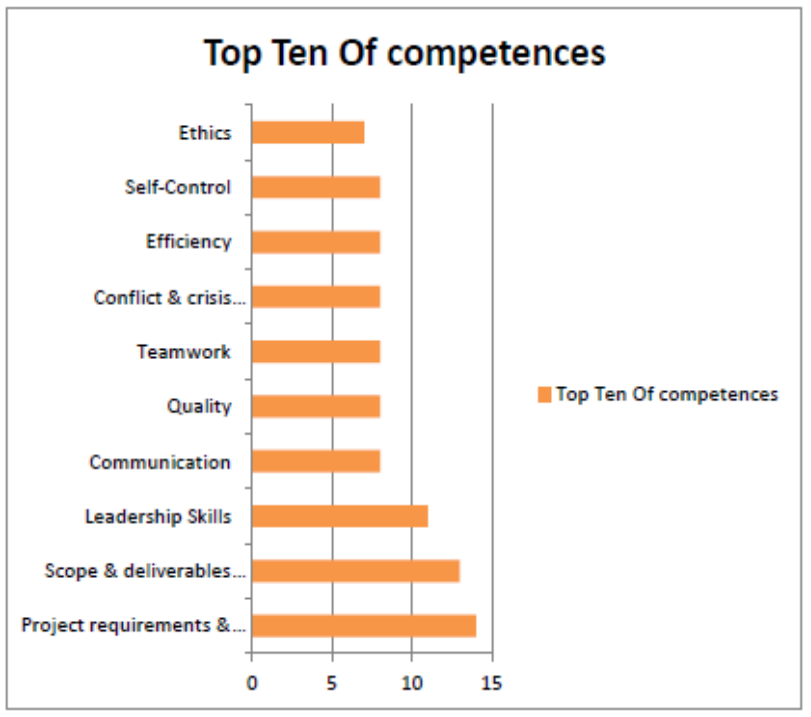

Figure 6. Rating of the top teen competences
The categorization of soft and hard skills does not reflect the technical and behavioral competences directly in the Eye of Competences. Among the ten competences that respondents of the survey ranked the highest, three are defined in the Eye as technical, but would fall under soft competences in the definition used in this paper, i.e. project management, communication and teamwork.

The decision to let participants rate the competence rather than prioritize them may have reduced the possibility of seeing marked differences between competences. A better method would have been to use the pilot survey to get top competences and let the participants in the main survey prioritize them. Lack of trust scores lower than in most studies on virtual teams. One possible explanation is that the participants of this study all work in the IT projects, although in distributed teams.

TABLE I.

SHOWING CATEGORIZATION ON TECHNICAL/BEHAVIORAL AND SOFT/HARD COMPETENCES

\begin{tabular}{|l|l|l|}
\hline Competences & T /B & H / S \\
\hline requirements \& objectives & T & H \\
\hline Scope \& deliverables are well defined & T & H \\
\hline Leadership Skills & B & S \\
\hline Communication & T & S \\
\hline Quality & T & H \\
\hline Teamwork & T & S \\
\hline Conflict \& crisis handling & B & H \\
\hline Efficiency & B & S \\
\hline Self-Control & B & S \\
\hline Ethics & B & S \\
\hline
\end{tabular}

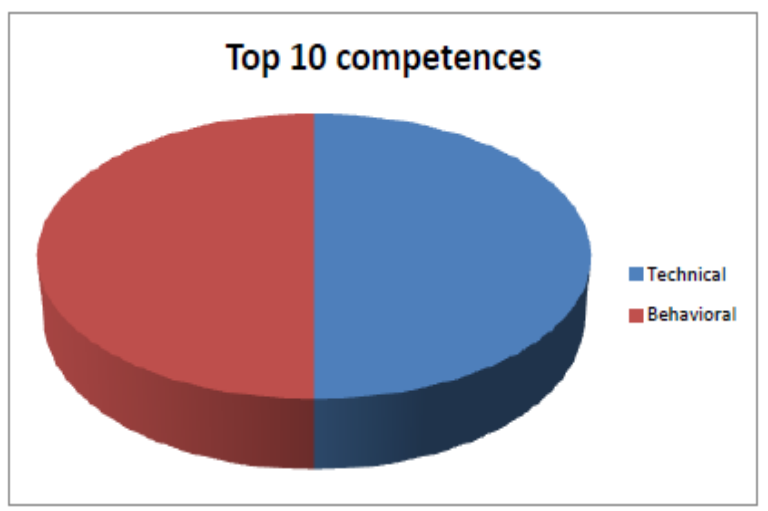

Figure 7. Top 10 divided by Technical or Behavioral Competences

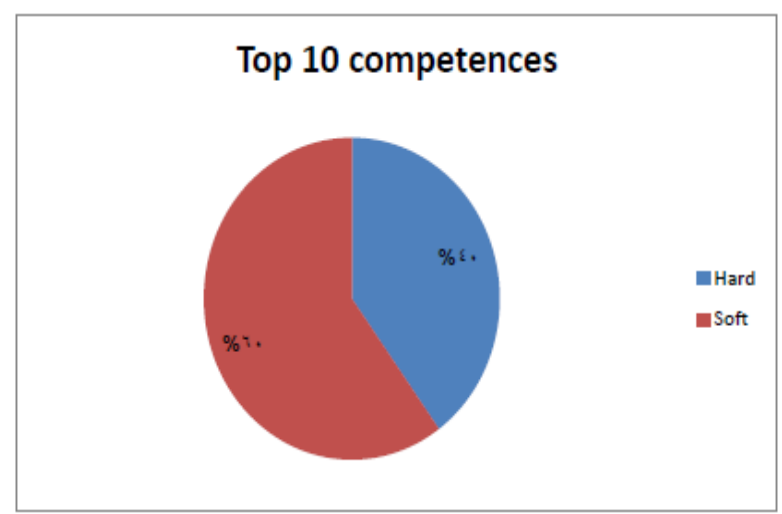

Figure 8. Top 10 divided by Hard or Soft competences 
The fact that competences such as requirements, objectives, scope and deliverables are scoring high is consistent with other studies. The authors in [12] summarized it in the following words: "Virtualism requires a rather more robust and clear view of exactly what outcomes are required. The finding that virtual team members found time zone differences and language difference challenging is also consistent with other studies such as the study. Perhaps a new competence needs to be added to the Eye of Competence, one that focuses on virtual work processes, since globalization is spreading at a fast rate, and companies are increasingly using distributed teams as part of their daily business [12].

\section{CONCLUSION}

From both the survey's findings and the literature review it can be stated that both soft and hard skills are needed for the optimal IT project manager to lead a virtual team. To be successful in this area it is not sufficient to be outstanding in technical competences. Soft skills like communication, teamwork and leadership are just as important. Even though the research was confined to only one software company it should have given some indication as to what is needed to manage an IT project using virtual teams. Future studies are needed that would reach across the IT industry where globally distributed teams would be further investigated as well as their leadership. To summarize, the most important competence for leading successful a virtual IT team are:

- The team has to be able to see the whole picture / Clarity of objectives

- Leadership/Project management

- Communication

- Reliability/Efficiency

- Teamwork

- Quality Management

\section{ACKNOWLEDGMENT}

The authors acknowledge the academic support received from Arab East Colleges for Graduate Studies and from Educity center for Learning and Scientific Research, as well as from Cairo Higher Institute for Computer Science, Engineering and Managements.

\section{REFERENCES}

[1] Conchúir, E. Ó., Holmström, H. O., Ågerfalk, P. J., \& Fitzgerald, B. (2008). Benefits of global software development: the known and unknown. International conference on software process, ICSP. Leipzig.

[2] (Mario Eberlein Cultures as critical success factor for successful global project management in multi-national IT projects Journal of Information Technology Management Journal of Information Technology Management Volume XIX, Number 3, 2008.)

[3] (Asta Hildur Asolfsdottir Leading a Virtual Team: Globalization and IT Project Management May 2012)

[4] (Lowell C. Matthews and Bharat Thakkar ; The Impact of Globalization on Cross-Cultural CommunicatioN ;The 2nd KoreaAmerica Student Conference 2012)
[5] (Ásgeir Haukur Guðmundsson What are the success factors for project leaders in virtual teams? 2012

[6] (Oyeyemi Kayode Impact of Globalization on Human Resource Management," Science Journal of Business Management, Volume 2012)

[7] A Guide to the Project Management Body of Knowlwdge PMBOK Guide fourth Edition. (2008). Pennsylvania: Project Management Institute Inc.

[8] Maznevski, M. L., \& Chudoba, K. M. (2000). Bridging Space Over Time: Global Virtual Team Dynamics and Effectiveness. Organization Science, 473-492. http://dx.doi.org/10.1287/ orsc.11.5.473.15200

[9] Binder, J. (2007). Global Project Management: Communication, Collaboration and Management Across Borders. Hampshire: Gower Publishing Limited.

[10] Zakaria, N., Amelinckx, A., \& Wilemon, D. (2004, March). Working Together Apart? Building a Knowledge-Sharing Culture for Global Virtual Teams. Creativity and Innovation Management, 15-29. http://dx.doi.org/10.1111/j.1467-8691.2004.00290.x

[11] Egeland, B. (2010, February 26). Virtual Teams: Key Success Factors - Part 3. Retrieved April 15, 2012, from Project Management Tips guidance for real life situations: http://pmtips.net/virtual-teams-key-success-factors-part-3/

[12] Smith, A., \& Sinclair, A. (2003). What makes a excellent virtual manager. Roffey Park.

[13] Schwalbe, K. (2007). Information Technology Project Management 5th edn. Boston, USA: Course Technology.

[14] Ashish, A., \& Gambardella, A. (2005). The globalization of the software industry: perspectives and opportunities for developed and developing countries. Innovation policy and the economy, 133.

[15] Ebert, C., Alcatel, A., \& De Neve, P. (2001). Surviving global software development. Software, IEEE, 62-69. http://dx.doi.org/10.1109/52.914748

[16] Joseph, D., Ang, S., Chang, H. R., \& Slaughter, S. A. (2010). Practical Intelligence in IT: Assessing Soft Skills of IT Professionals. Communications of the ACM, 149-154. http://dx.doi.org/10.1145/1646353.1646391

[17] http://ipma.ch/resources/ipma-publications/ipma-competencebaseline/

\section{AUTHORS}

Dr. Hosam F. El-Sofany received his Ph.D. and his M. Sc. degree in Computer Science. He is currently an Assistant Professor of CS at Cairo Higher Institute, and Director of Educity Center for Learning and Scientific Research, Cairo, Egypt. He has a strong technical background including the development of Web-based and Mobile-based educational systems. His research interest include: E-Learning, M-Learning, XML Databases, Databases Systems, and Semantic Web. Dr. El-Sofany's e-mail is: hosam_elsofany@hotmail.com.

Hassan M. Alwadani, Student in MSc program at Arab East Colleges for Graduate Studies, Department of Computer Science, Riyadh, Kingdom of Saudi Arabia.

Amer Alwadani, Student in MSc program at University of Dayton, Department of Computer Science, Ohio State, USA.

Submitted 08 July 2014. Published as resubmitted by the authors 08 December 2014. 